\title{
Norms on Quotient Spaces of The 2-Inner Product Space
}

\author{
Harmanus Batkunde \\ Algebra and Analysis Division, Department of Mathematics, Pattimura University \\ e-mail: h.batkunde@fmipa.unpatti.ac.id
}

\begin{abstract}
This paper discussed about construction of some quotients spaces of the 2-inner product spaces. On those quotient spaces, we defined an inner product with respect to a linear independent set. These inner products was derived from the $n$-inner product. We then defined a norm which induced by the inner product in these quotient spaces.
\end{abstract}

Keywords: $n$-inner product spaces, norm, quotient space.

\section{Introduction}

The concept of the inner product space

In 1960's S. Gähler initially introduced the concept of 2-normed space [1-4] which is a generalization of the concept of norm space. Let $X$ be a real vector space of dimension $d \geq 2$. A real-valued function $\|\cdot, \cdot\|$ on $X \times X$ that satisfies the following conditions:

N1. $\|x, y\|=0$ if and only if $x$ and $y$ are linearly dependent, for all $x, y \in X$;

N2. $\|x, y\|=\|y, x\|$, for all $x, y \in X$;

N3. $\|\alpha x, y\|=|\alpha|\|x, y\|$ for all $\alpha \in \mathbb{R}$ and $x, y \in X$;

N4. $\|x, y+z\| \leq\|x, y\|+\|x, z\|$; for all $x, y, z \in X$;

is called a 2-norm on $\mathrm{X}$, and the pair of $(X,\|\cdot \cdot\|)$ is called a 2 -normed spaces.

For example, if $(X,\langle\cdot\rangle$,$) is an inner product space then the following function$

$$
\|x, y\|=\left|\begin{array}{ll}
\langle x, x\rangle & \langle x, y\rangle \\
\langle y, x\rangle & \langle y, y\rangle
\end{array}\right|^{\frac{1}{2}}
$$

is a 2-norm in $X$. We can check that the above function satisfies (N1-N4). Geometrically, he value of $\|x, y\|$ represents the area spanned by $x$ and $y$. We called it standard 2-norm on $X$.

Moreover, one can see that from (N3) and (N4) we have 


$$
\|x, y\| \geq 0 \text {, for any } x, y \in X
$$

which means the 2-norm is nonnegative. On 2-normed space, we also have

$$
\|x, \alpha x+y\|=\|x, y\| \text {, for any } \alpha \in \mathbb{R} \text { and } x, y \in X .
$$

A concept which is related to the 2-normed space is the 2-inner product space. This is a generalization of the concept of inner product space. Concept of 2-normed space and 2inner product space it has been developed extensively with various results by many researchers, (see for instance [5-11]).

Let $X$ be a real vector space and $\operatorname{dim}(X)=d \geq 3$. A function $\langle\cdot, \cdot \mid \cdot\rangle: X \times X \times X \rightarrow \mathbb{R}$ which satisfies the following conditions

I1. $\langle x, x \mid z\rangle \geq 0$ and $\langle x, x \mid z\rangle=0$ if and only if $x$ and $z$ are linearly dependent;

I2. $\langle x, x \mid z\rangle=\langle z, z \mid x\rangle$, for any $x, z \in X$;

I3. $\langle x, y \mid z\rangle=\langle y, x \mid z\rangle$, for any $x, y, z \in X$;

I4. $\langle\alpha x, y \mid z\rangle=\alpha\langle x, y \mid z\rangle$, for any $\alpha \in \mathbb{R}$ and $x, y, z \in X$;

I5. $\left\langle x_{1}+x_{2}, y \mid z\right\rangle=\left\langle x_{1}, y \mid z\right\rangle+\left\langle x_{2}, y \mid z\right\rangle$;

is called a 2-inner product on $X$, while the pair $(X,\langle\cdot, \cdot \mid \cdot\rangle)$ is called a 2-inner product space.

We can derive a 2-norm from a 2-inner product by define

$$
\|x, z\|=\langle x, x \mid z\rangle^{\frac{1}{2}} .
$$

We say that the 2-norm in (1) is induced by a 2-inner product.

Furthermore, we will construct some quotient spaces in a 2-inner product space and define an inner product in each quotient space. If $X$ be a vector space, then we can construct some quotient spaces of $X$. These quotient spaces can be constructed with respect to some equivalent relations. Moreover, we can define an inner product in $X$ whenever it is possible.

Let $\sim$ be an equivalence relation on $X$. For an $x \in X$, the set of all elements equivalent to $x$ is denoted by

$$
\bar{x}:=\{y \in X: y \sim x\},
$$


is called the equivalence class of $x$.

Let $X$ be a vector space over $F, x \in X$, and $V \subseteq X$. The set $\bar{x}=x+V=\{x+v: v \in V\}$ is called a coset of $V$ in $X, x$ is called a coset representative for $x+V$. Moreover, the set of all cosets of $V$ on $X$ is denoted by

$$
X / V=\{\bar{x}: x \in X\} .
$$

This set is called the quotient of $X$ modulo $V$. [12]

An addition and scalar multiplication operation on $X / V$ is defined by $\bar{x}+\bar{y}=\overline{x+y}$ and $\alpha \bar{x}=\overline{\alpha x}$ respectively, for any $x, y \in X / V$ and $\alpha \in F$.

\section{Results and Discussion}

Now, we will construct some quotient spaces in a 2-inner product space. Let $(X$,$) be a 2-$ inner product space and $Y=\left\{y_{1}, y_{2}\right\}$ be a linear independent set on $X$. Define a set of $X$ generated by $\left\{y_{1}\right\}$

$$
Y_{1}=\operatorname{span}\left\{y_{1}\right\}=\left\{\alpha y_{1}: \alpha \in \mathbb{R}\right\}
$$

It is easy to see that $Y_{1}$ is a subspace. For any $x \in X$, the corresponding coset of $Y_{1}$ on $X$ is

$$
\bar{x}=\left\{x+\alpha y_{1}: \alpha \in \mathbb{R}\right\}
$$

Then we have $\overline{0}=\operatorname{span}\left\{y_{1}\right\}=Y_{1}$, and if $\bar{x}=\bar{y}$ then $x-y \in Y_{1}$. Further, we define the quotient space of $X$ as

$$
X_{1}^{*}=X / Y_{1}=\{\bar{x} \quad: \quad x \in X\}
$$

The addition and scalar multiplication also apply on $X_{1}^{*}$, those are

1. $\bar{x}+\bar{y}=\overline{x+y}$, for for any $x, y \in X / Y_{1}$;

2. $\alpha \bar{x}=\overline{\alpha x}$ respectively, for any $x \in X / Y_{1}$ and $\alpha \in \mathbb{R}$.

Next, on $X_{1}^{*}$ we define a function $\langle\cdot, \cdot\rangle_{1}: X_{1}^{*} \rightarrow \mathbb{R}$ defined by 


$$
\langle\bar{x}, \bar{y}\rangle_{1}=\left\langle x, y \mid y_{1}\right\rangle
$$

We can see easily that he function defined on (3) is well defined. This function defines an inner product on $X_{1}^{*}$, so that the pair $\left(X_{1}^{*},\langle\cdot,\rangle_{1}\right)$ is an inner product space as stated in the following theorem.

Theorem 2.1. Let $(X,\langle\cdot, \cdot \mid \cdot\rangle)$ be a 2-inner product space and $Y=\left\{y_{1}, y_{2}\right\}$ is a linearly independent set in $X$. Then $\left(X_{1}^{*},\langle\cdot, \cdot\rangle_{1}\right)$ is an inner product space, where $\langle\cdot, \cdot\rangle_{1}$ is a function in (3).

\section{Proof.}

To prove the above theorem we have to prove that the function we defined in (2) is an inner product on $X_{1}^{*}$. Recall that

$$
\langle\bar{x}, \bar{y}\rangle_{1}=\left\langle x, y \mid y_{1}\right\rangle
$$

then using the properties of 2-inner product (N1) we have

$$
\langle\bar{x}, \bar{x}\rangle_{1}=\left\langle x, x \mid y_{1}\right\rangle \geq 0
$$

If $\langle\bar{x}, \bar{x}\rangle_{1}=0$, then $\left\langle x, x \mid y_{1}\right\rangle=0$. This means $x$ and $y_{1}$ are linearly dependent. We can write $x=\alpha y_{1}$, for an $\alpha \in \mathbb{R}$ or $x \in \operatorname{span}\left\{y_{1}\right\}=Y_{1}$, which means $\bar{x}=\overline{0}$. Conversely, if $\bar{x}=\overline{0}$ then it is obvious that $\langle\bar{x}, \bar{x}\rangle_{1}=\langle\overline{0}, \overline{0}\rangle_{1}=\left\langle 0,0 \mid y_{1}\right\rangle=0$.

Next, using (N3) and (N4) we have for any $\alpha \in \mathbb{R}$ and $x, y \in X$, we have

$$
\langle\bar{x}, \bar{y}\rangle_{1}=\left\langle x, y \mid y_{1}\right\rangle=\left\langle x, y \mid y_{1}\right\rangle=\langle\bar{y}, \bar{x}\rangle_{1}
$$

and

$$
\langle\alpha \bar{x}, \bar{y}\rangle_{1}=\alpha\left\langle x, y \mid y_{1}\right\rangle=\alpha\langle\bar{x}, \bar{y}\rangle_{1}
$$

Moreover, using (N5)we have for any $x_{1}, x_{2} \in X$

$$
\left\langle\overline{x_{1}}+\overline{x_{2}}, \bar{y}\right\rangle_{1}=\left\langle x_{1}+x_{2}, y \mid z\right\rangle=\left\langle x_{1}, y \mid z\right\rangle+\left\langle x_{2}, y \mid z\right\rangle=\left\langle\overline{x_{1}}, \bar{y}\right\rangle_{1}+\left\langle\overline{x_{2}}, \bar{y}\right\rangle_{1} .
$$

These prove that the function on (3) is an inner product space on $X_{1}^{*}$, which means $\left(X_{1}^{*},\langle\cdot, \cdot\rangle_{1}\right)$ is an inner product space. 
Recall that if $(X,\langle\cdot, \cdot\rangle)$ is an inner product space then the following formula

$$
\|x\|=\langle x, x\rangle^{\frac{1}{2}}
$$

defines a norm on $X$, where $x \in X$. By this fact, we can define a norm on $X_{1}^{*}$.

Let $(X,\langle\cdot, \cdot \mid \cdot\rangle)$ be a 2-inner product space and $Y=\left\{y_{1}, y_{2}\right\}$ is a linearly independent set in $X$. A function $\|\cdot\|_{1}: X_{1}^{*} \rightarrow \mathbb{R}$ which defined by

$$
\|x\|_{1}=\langle x, x\rangle_{1}^{\frac{1}{2}}
$$

is a norm on $X_{1}^{*}$.

By (3) and (1) we can see that

$$
\langle x, x\rangle_{1}^{\frac{1}{2}}=\left\langle x, x \mid y_{1}\right\rangle^{\frac{1}{2}}=\left\|x, y_{1}\right\|
$$

This means The norm $\|\cdot\|_{1}$ defined in (4) can be seen as 2-norm on $X$ with respect to $y_{1}$.

Furthermore, we will construct another quotient space in a 2-inner product space. Let $(X$,$) be a 2-inner product space and Y=\left\{y_{1}, y_{2}\right\}$ be a linear independent set on $X$. For constructing the previous quotient space we considered $y_{1} \in Y$. Now, we consider $y_{2} \in$ $Y$ and use the same construction to define another quotient space. Define a set of $X$ generated by $\left\{y_{2}\right\}$

$$
Y_{2}=\operatorname{span}\left\{y_{2}\right\}=\left\{\alpha y_{2}: \alpha \in \mathbb{R}\right\}
$$

The set $Y_{1}$ is a subspace and for any $x \in X$, the corresponding coset of $Y_{2}$ on $X$ is

$$
\bar{x}=\left\{x+\alpha y_{2}: \alpha \in \mathbb{R}\right\}
$$

Then we have $\overline{0}=\operatorname{span}\left\{y_{2}\right\}=Y_{2}$, and if $\bar{x}=\bar{y}$ then $x-y \in Y_{2}$. Further, we define the quotient space of $X$ as 


$$
X_{2}^{*}=X / Y_{2}=\{\bar{x} \quad: \quad x \in X\}
$$

The addition and scalar multiplication also apply on $X_{2}^{*}$.

On $X_{1}^{*}$ we define a function $\langle\cdot, \cdot\rangle_{2}: X_{2}^{*} \rightarrow \mathbb{R}$ defined by

$$
\langle\bar{x}, \bar{y}\rangle_{2}=\left\langle x, y \mid y_{2}\right\rangle
$$

This function defines an inner product on $X_{2}^{*}$, so that the pair $\left(X_{2}^{*},\langle\cdot,\rangle_{2}\right)$ is an inner product space as stated in the following theorem.

Theorem 2.2. Let $(X,\langle\cdot, \mid \cdot\rangle)$ be a 2 -inner product space and $Y=\left\{y_{1}, y_{2}\right\}$ is a linearly independent set in $X$. Then $\left(X_{2}^{*},\langle\cdot, \cdot\rangle_{2}\right)$ is an inner product space, where $\langle\cdot, \cdot\rangle_{2}$ is a function in (6).

\section{Proof.}

The proof is analogue with the proof of Theorem 2.1.

As an analogue result, we also have the following formula

$$
\|x\|_{2}=\langle x, x\rangle_{2}^{\frac{1}{2}} ; x \in X
$$

defines a norm on $X_{1}^{*}$.

By (6) and (1) we can see that

$$
\langle x, x\rangle_{2}^{\frac{1}{2}}=\left\langle x, x \mid y_{2}\right\rangle^{\frac{1}{2}}=\left\|x, y_{2}\right\|
$$

This means The norm $\|\cdot\|_{2}$ defined in (4) can be seen as 2-norm on $X$ with respect to $y_{2}$.

On [13], Gunawan derived an inner product on an $n$-inner product space. The inner product is derived from the $n$-inner product. Let $(X,\langle\cdot, \cdot \mid \cdot, \ldots\rangle$,$) and Y=\left\{y_{1}, \ldots, y_{n}\right\}$ be a linearly independent set. Gunawan derived an inner product on $X$, defined by

$$
\langle x, y\rangle=\sum_{\left\{i_{1}, \ldots, i_{n}\right\} \subset\{1, \ldots, n\}}\left\langle x, y \mid y_{i_{1}}, \ldots, y_{i_{n}}\right\rangle .
$$


For $n=2$, the above formula can be written as

$$
\langle x, y\rangle=\left\langle x, y \mid y_{1}\right\rangle+\left\langle x, y \mid y_{2}\right\rangle
$$

As we can see, each term of right hand side of equation (8) is the formula of inner product on two quotient spaces we constructed earlier.

$$
\langle x, y\rangle=\langle x, y\rangle_{1}+\left\langle x, y_{2}\right\rangle_{2}
$$

As a consequence, using both of inner products we constructed earlier we can investigate some aspects the inner products of both space. Moreover, the norm induced by (9) can be written as

$$
\|x\|=\langle x, y\rangle^{\frac{1}{2}}=\left(\langle x, y\rangle_{1}^{2}+\left\langle x, y_{2}\right\rangle_{2}^{2}\right)^{\frac{1}{2}}=\left(\|x\|_{1}^{2}+\|x\|_{2}^{2}\right)^{\frac{1}{2}} .
$$

On the above norm, we also can see the induced norms on two quotient spaces we constructed on the right hand side of equation (10).

Furthermore, we can use these inner products or the induced norms to observe some aspects on an $n$-inner product space.

\section{References}

[1] Gähler, S., 1964, Lineare 2-normierte Räume, Math. Nachr. 28, 1-43.

[2] Gähler, S., 1969, Untersuchungen über verallgemeinerte m-metrische Räume. I, Math. Nachr. 40, 165-189

[3] Gähler, S., 1969, Untersuchungen über verallgemeinerte m-metrische Räume. II, Math. Nachr. 40, 229-264

[4] Gähler, S., 1969, Untersuchungen über verallgemeinerte m-metrische Räume. III, Math. Nachr. 41, 23-36 (German). MR 40\#1989. Zbl 182.56601.

[5] Batkunde, H., \& Gunawan, H., 2019, On the topology of n-normed spaces with respect to norms of its quotient spaces, Adv. Stud. Contemp., 29, 89-98.

[6] Gunawan, H., \& Mashadi., 2001, On n-normed spaces, Int. J. Math. Math. Sci. 27, 631-639.

[7] Konca, S., \& Idris, M., 2015, Equivalence among three 2-norms on the space of psummable sequences, Journal of Inequalities and Special Functions, 7(4), 218-224.

[8] Huang, X., \& Tan, A., 2018, Mapping of preserving n-distance one in n-normed spaces, Aequationes Math. 92, 401-413.

[9] Batkunde, H., Gunawan, H., \& Neswan, O., 2018, n-Normed spaces with norms of its quotient spaces, J. Phys.: Conf. Ser. 1097, 012079. 
[10] Najmabadi, B. M., Shateri, T. L., \& Sadeghi, G., 2020, A Type of Orthonormal Bases on 2-*-Inner Product Spaces, Studia Scientiarum Mathematicarum Hungarica, 57(4), 541-551.

[11] Sababe, S. H., 2020, On 2-inner product spaces and reproducing property, The Korean Journal of Mathematics, 28(4), 973-984.

[12] Roman, S., 2005, Advance Linear Algebra. Springer: New York, pp 87-89.

[13] Gunawan, H., 2002, Inner products on n-inner product spaces. Soochow Journal of Mathematics, 28(4), 389-398. 